\title{
Electrohydrodynamic Effects on Characteristic of Isolated bubbles in the nucleate Pool Boiling regime.
}

\author{
J. Madadnia, \& H. Koosha, \\ Faculty of Engineering, University of Technology, Sydney (UTS), \\ PO Box 123, NSW 2007, Australia \\ Email: J.Madadnia@uts.edu.au, \\ URL:http://www.eng.uts.edu.au/ jafarm
}

\section{Abstract}

Application of electric fields demonstrates great promise as an active heat transfer enhancement technique. Here, we study experimentally some characteristics of isolated bubbles such as bubble departure diameter, nucleation rate (frequency), and density of nucleation sites under the influence of an electric field In the nucleate pool boiling regime, isolated bubbles were recorded with a high-speed video camera at a rate of 1000 frames per second.

The quantitative and qualitative analyses of bubble characteristics, and latent heat transfer concluded that at a constant heat flux condition in "the isolated bubble regime", the "density of nucleation sites" was a very sensitive boiling parameter when electric field varied. The latent heat contribution varied proportional with the density of nucleation sites when heat flux and/or electrode voltage varied. This further simplifies the complex task of modeling of the EHD enhanced pool boiling heat transfer in the isolated bubble regime.

\section{Keywords:}

Electrohydrodynamic (EHD), Nucleate pool boiling, high-speed video camera, heat transfer enhancement, bubble characteristics.

\section{Introduction}

An active enhancement of heat transfer by electrohydrodynamic (EHD) is very important to many industries including heat exchanger manufacturing, and power generation. EHD has recently received a great deal of attention from many researchers. EHD can be explained by the classic theory of electricity and magnetism. The theory suggests that the "Maxwell stresses" develop body forces in a fluid, leading to localized fluid motions [1-15]. This electroconvection can improve heat transfer coefficient by several order of magnitudes, and at a constant heat flux condition can suppress nucleation. Because of the complexity of the EHD phenomena involved, great efforts have been made on the fundamental mechanisms of EHD effects on bubble dynamics [Ogata and Yabe 1991, 1993, Kweon et al. 1996, 1998, Kweon and Kim 2000, Nguyen and Madadnia 2000(a,b)]. It has been found from those studies that a nonuniform electric field influences bubble dynamics and boiling heat transfer coefficient. Nguyen and Madadnia (2000a,b) studied the behavior of isolated nucleated bubbles at low heat fluxes. They noticed that bubbles departure diameter gradually decrease until nucleation is suppressed and a single phase region is formed when electric field strengths exceeded a certain threshold value. This single phase electroconvection region has a very high heat transfer capability. Similarly, Kweon and Kim, 2000 studied EHD effects at high heat fluxes between a heated wire and an horizontal electrode and observed that bubbles were 
organized in more ordered columns with smaller diameters and higher departure frequencies, with reduced coalescence when the electrode voltage exceeded a certain threshold value.

Despite significant effects of EHD on the bubble dynamics, limited information is available on EHD effects on size, shape, growth rate, density of nucleation sites, and latent heat transfer contribution of bubbles. Moreover, no theory exist yet for EHD-enhanced boiling concerning the relationship between nucleate boiling heat transfer and the boiling dynamics in a nonuniform electric field. Since the information about bubble dynamics is not well understood, detailed knowledge on bubble characteristics is needed to clarify the basic mechanisms of the EHD boiling process. Particularly, the enhancing effects of an electric field on the nucleate boiling heat transfer can be well explained in terms of bubble behaviors

In this experimental investigation, the effects of an electric field on the characteristics of isolated bubbles in the nucleate pool boiling regime are studied. For this purpose, the bubble departure size, frequency of nucleation, and density of nucleate sites were measured at low heat fluxes and high electric fields, using a very high speed video camera. These are used to determine how the relative latent heat contribution is affected by an electric field.

\section{Experimental Apparatus}

\subsection{Experimental setup}

A schematic diagram of the EHD pool boiling apparatus is shown in Fig.1. The apparatus consisted of a double-glass-windowed-horizontal cylindrical boiling chamber of 25 $\mathrm{cm}$ in diameter, and $25 \mathrm{~cm}$ in length, an AC-power supply to an electrically heated wire, a DC power supply to charge a single electrode wire, a condenser coil, Voltmeters, Ammeters, and thermocouples. The boiling chamber also housed a grounded electrically heating Cr80Ni20wire (-ve) of, $85 \mathrm{~mm}$ long, $1 \mathrm{~mm}$ diameter, positioned $5 \mathrm{~mm}$ apart from an energized horizontal copper wire (+ve), $1.6 \mathrm{~mm}$ in diameter, and.85.0 $\mathrm{mm}$ in length, and in parallel. During experiment, the pressure and the saturation temperature were kept constant and Data were taken at steady state conditions.

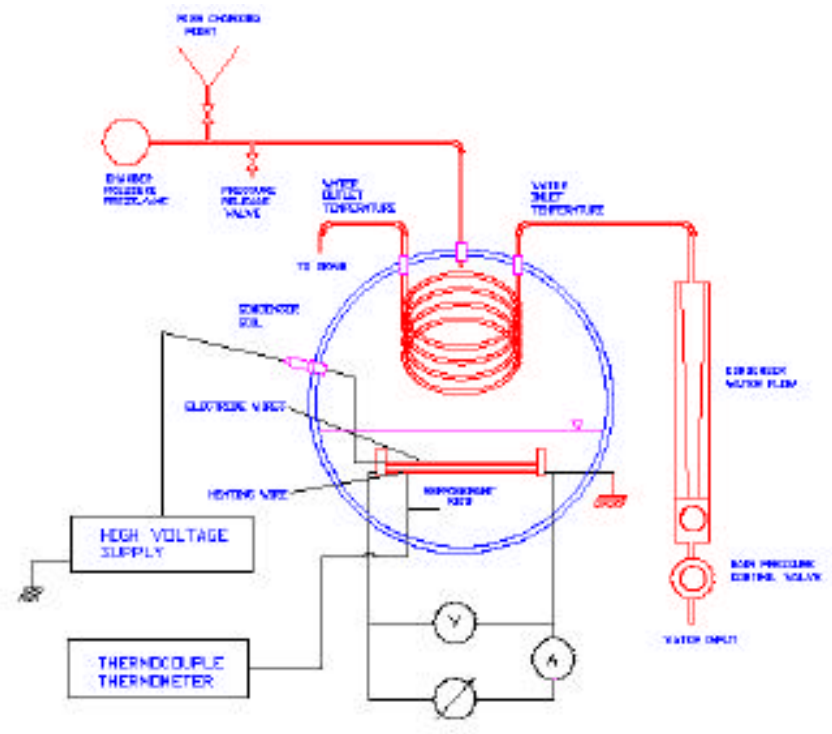

Figure 1: Schematic diagram of the experimental setup 


\subsection{Experimental Procedures}

Acetone was used to clean thoroughly the boiling chamber, the condenser coil, and the heated and electrode wire assembly. The dielectric liquid R123 was selected as the working fluid for its low boiling point and its environmentally friendly character. During experiments, the lower half of the chamber, where the wires were positioned, was filled with the refrigerant. The condenser coil was positioned at the top half of the chamber. The mass flow rate, inlet and outlet temperatures of the coolant water flowing through the condensing coil were measured using a rotameter and thermocouples.

The rig was degassed and vacuumed before filling the lower half of the chamber with refrigerant R123. The mass flow rate, inlet and outlet temperatures of the coolant water flowing through the condensing coil were measured using Rotameters and thermocouples on the top of the boiling cylinder.

Voltage and current from an ac-power supply unit to the heated wire were measured and the corresponding heat flux is calculated. The saturated liquid temperature at the atmospheric pressure is measured by a k-type submerged thermocouple in the liquid, so that heat losses to surrounding are kept constant in all runs.

The electric conductivity $(\sigma=25 \mathrm{nS} / \mathrm{m})$ and the electric permittitivity $(\varepsilon=44 \mathrm{pF} / \mathrm{m})$ of R123 were measured experimentally and found to be in the appropriate range for the saturated liquid at the atmospheric pressure, as suggested by Turnbull (1968 a,b), and Seyed-Yagobi et al (1996).

At the start of each test, R123 was heated to its saturation temperature at atmospheric pressure. The ac-power to the heating wire increased to incept bubbles nucleation. The start of nucleation was always abrupt and bubbles usually covered almost the entire wire. The heat flux was therefore gradually reduced by controlling the heater voltage. Accurate determination of bubble characteristics were very difficult for coalescent bubbles. Therefore, the region of isolated nucleated bubbles was investigated at this experiment. Isolated nucleation sites were formed at heat fluxes ranging from $3.7 \mathrm{~kW} / \mathrm{m}^{2}$ to $40 \mathrm{~kW} / \mathrm{m}^{2}$. Bubbles jointed together and coalesced at around $40 \mathrm{~kW} / \mathrm{m}^{2}$. At each heat flux, electrode voltage was increased until all nucleated bubbles disappeared. However, it was noticed that in the coalesced bubble regime with a very high heat flux, increasing electrode voltage either led to short circuits, or formation of higher frequency smaller bubbles, and suppression of nucleation was not observed.

The high speed video camera was used to record nucleation at a rate approaching 1000 frames per second and up to 5000 frames were taken from each run. At a viewing angle perpendicular to the plane passing the wires plane, the projected wire diameter was measured. A relative size scale was then obtained by comparing it with the actual wire diameter. The projected bubble diameters were also measured and the relative scale was used to quantify the actual bubble diameters. 


\section{Results And Discussion}

\subsection{Qualitative analysis}

The high-speed video camera was used to record nucleated bubbles at several intermediate heat fluxes ranging from 3.7 to $40 \mathrm{~kW} / \mathrm{m}^{2}$. The bubble characteristics such as departure diameter, frequency, and density of nucleation sites, were measured from each frame and the average values were plotted for the intermediate heat fluxes and electrode voltages. Typical high-speed video pictures from instantaneous behaviors of bubbles are shown in Figure 2a for the three heat fluxes; 3.7, 8.0, and $12.1 \mathrm{~kW} / \mathrm{m}^{2}$ near the minimum superheat in the nucleate pool boiling regime. It is observed that bubble size and frequency of nucleation increased with increasing heat flux at the zero electric field. However, frequency and size remained constant in the isolated bubble regime.

When a typical heat flux of $35.4 \mathrm{~kW} / \mathrm{m}^{2}$ was kept constant, electrode voltage was increased until all nucleate bubbles disappeared. Bubble size and frequency increased slightly and then remained unchanged until a threshold electrode voltage of $6 \mathrm{kV}(120 \mathrm{kV} / \mathrm{cm})$ was applied, and then decreased at higher voltages. Figs $2 \mathrm{~b}$ show three typical high-speed camera pictures from bubbles at three electrode voltages $(6,6.6$, and $7,5 \mathrm{kV})$ indicating that the imposing electric field reduced bubble size, and frequency of nucleation. Nucleation ceased at $8 \mathrm{kV}(160 \mathrm{kV} / \mathrm{cm})$ and beyond where no further cooling on wire was observed. Similar observations were also reported by Baboi et al (1968), and most recently by Kweon and Kim (2000).

In the absence of electric fields, bubbles depart nucleation sites when buoyancy forces exceed the surface tension forces. However, when electric field is applied, theoretically both the Coulomb and polarization forces acted on the bubbles. Fluid relaxation time determines the time that detached bubbles hold charges and consequently are influenced by the Coulomb force. Since bubbles were negatively charged due to their attachment to the heated wire, the Coulomb force pulled the bubbles away from the heating surface towards to the energized electrode. The contribution of Coulomb force is small due to the fact that gas bubbles in a charged pure liquid do not transport charge [Babiot et al (1964)].

At the same time, Electric conductivity of R123 increases and its electric permittivity decreases with temperature. Therefore EHD generated a weaker electric field in the superheated liquid region which was closer to the heated wire, than that in the saturated temperature region. Consequently, bubbles were pushed on the heated wire, leading to increasing bubble size, and frequency. This polarization force (dielectrophoresis) induced motion of bubbles which were attracted towards a region of weaker electric field which was closer the heated wire. This permitivity gradient force pressed the bubble against the heating surface. The relative strength of these two forces depends on the strength of electric field, the electrode design, and the properties of R123. This was also reported by Ogata and Yabe (1991, 1993) who reported that bubbles were suppressed on the heated surface when electric field at lower part of the bubble was weaker than the that at the upper part of the bubble. Seyed-Yagoobi and Bryan (1999) also noticed that that nonuniformity of electric field distribution around the heating surface pushed the bubbles around the cylindrical heated surface.

At low heat fluxes, and high electric fields, small number of bubbles departed from the wire before they completely disappear, as we can see from Figures $2(a, b)$ which are similar to 
observations made by Ogata and Yabe (1991, 1993). They noticed that heat transfer enhancement became maximum when the fluid relaxation time $((\tau=\varepsilon / \sigma=$ for dielectrics is in the order of $1 \mathrm{~ms}$ and for R123 in this experiment was $1.76 \mathrm{~ms}$ ) is less than the characteristic time of bubble detachment from the surface $(\tau=1 / \mathrm{f}=1 / 100 \mathrm{~s}=10 \mathrm{~ms})$.

At a wire and a flat plate electrode configuration, Kweon and Kim (2000), noticed that, nucleated bubbles detached more rapidly from the heated surface and were attracted towards the opposite electrode when electrode voltage was increased.

The break up of bubbles at high electric fields (at a constant superheat) is an EHDinduced phenomenon and termed as Taylor-instability and also reported by [Ogata and Yabe 1991, 1993]. The following observations were made in the coalescence regime where bubbles completely covered the heating surface (beyond the isolated bubble regime); Density of active nucleation sites reached to its maximum and no additional nucleation site was developed when electrode voltage or heat flux varied. At a constant heat flux, frequency of bubble nucleation, and its departure diameter decreased with increase of electrode voltage as cooler liquid were induced around the heated wire.

Our visual observations indicated that the electric field modifies the bubble dynamics of the boiling process. At a constant heat flux of $35.4 \mathrm{~kW} / \mathrm{m} 2$, al electrode voltages below $6 \mathrm{kV}$, no appreciable effects of an electric field on bubble size, frequency, and density of nucleation sites were noticed. At above $6 \mathrm{kV}$, the bubble size, density, and frequency dropped significantly as the heated wire was cooled by the induced liquid.

\subsection{Quantitative analysis}

Experimental data were plotted in the following figures and quantitatively analyzed. Figures 3 and 4 show that bubble diameter and frequency of bubble nucleation were affected when electric voltage exceeded a threshold value of $6 \mathrm{kV}$ and heat flux exceeded $6 \mathrm{~kW} / \mathrm{m}^{2}$. Bubble diameters and frequency increased slightly relative to zero field conditions. It might be due to polarization forces which pushed bubbles on the heating surface, and kept them longer on the heating surface, leading to larger bubbles at departure. This also indicated that the polarization forces were slightly larger than the Coulomb forces, which pulled bubbles away from the surface. Bubble size and frequency remained unaffected by both electrode voltage and heat flux except at lower superheats near the inception of nucleation. EHD forces due to polarization forces, which pushed bubbles to the heating, surface leading to some deformation. Similarly at two constant heat fluxes of 13.2 and $35.4 \mathrm{~kW} / \mathrm{m}^{2}$, bubble sizes decreased only within the $20 \%$ to $30 \%$ of the maximum electrode voltage where superheat was low and finally bubbles disappeared completely. This is similar with observation of Baboi et al (1967) on R11 on a fine heating wire.

Figure 5 shows that density of bubble nucleation sites increased proportionally with the heat flux at zero field conditions. However, density of nucleation sites remained unchanged at both higher and moderate electric fields, and varied proportional with low electric fields at isolated region. At high electric fields bubbles shrunk and disappeared due to high cooling power of heated surface by the EHD-induced fluid. Figures 5 and 6 also show that latent heat contribution vary proportional with the heat flux, and inversely proportional with electrode voltage if the applied voltage is within the $20 \%$ to $30 \%$ of the maximum electrode voltage which suppressed nucleation completely.

Figure 6 shows that in the isolated bubble regime of nucleate pool boiling, density of 
nucleation sites and latent heat of boiling increased proportional with heat flux, and inversely proportional with the electrode voltage. The latent heat of boiling constituted less than $12 \%$ of the total heat flux and is controlled by the density of nucleation sites

\section{Conclusions}

Effects of electric field on bubble's departure diameter, frequency, and density of nucleation sites of isolated bubbles were analysed in the nucleate pool boiling regime. Electric fields and heat fluxes were varied. It was concluded that density of active nucleation sites played as an important bubble character which was very sensitive to variation of both heat fluxes and electric fields and varied proportional with the latent heat contribution. It is anticipate that this finding would further facilitate numerical and physical modeling of EHDenhanced pool boiling heat transfer.

\section{Acknowledgement}

The authors would like to thank TAMU for providing the opportunity of this research.

\section{References}

1. Baboi N. F., Bolaga M. K. and Klyukanov A.A., 1967, Some Features of Ebullition in an Electric Field, Report to All-Union Conference on Electrical Processing of Materials, Kiev.

2. Barthau, G., 1992, “Active Nucleation Site Density and Pool Boiling Heat Transfer- An Experimental Study,” Int. Jour. Of Heat and Mass Transfer, Vol. 35, pp. 271-278

3. Kweon, Y.C., Kim, M.H., Cho, H.J., Kang, I.S., \& Kim, S.J., 1996 "Effects of an electric field on the nucleate pool boiling and bubble behavior on a horizontal wire." In: Chen, J.C. (Ed.), Convective Flow Boiling. Taylor \& Francis, pp. 351-356.

4. Kweon, Y.C., Kim, M.H., Cho, H.J., \& Kang, I.S., 1998 "Study on the deformation and departure of a bubble attached to a wall in dc/ac electric fields." Int. J. Multiphase Flow $24,145-162$.

5. Kweon, Y.C., \& Kim, M.H. 2000 "Experimental study on nucleate boiling enhancement and bubble dynamic behavior in saturated pool boiling using a nonuniform dc electric field." Int. J. Multiphase Flow 26, 1351-1368.

6. Madadnia, J., and Nguyen, T. H., 2000" Effects of Parallel Electrodes on Electrohydrodynamically (EHD) Enhanced Boiling Heat Transfer" Proceeding of Symp. on Energy Engineering, in the 21th Century (SEE 2000), Ping Cheng (Ed), Begell House, N.Y, Wallingford (UK), Vol.1, pp. 349-354.

7. Nguyen, T, \& Madadnia J, 1999 "Modelling of Electrodynamically (EHD) Enhanced 
Boiling Heat Transfer using Helical Electrodes" Proceedings of SPIE, Design, Characterization, and Packaging for MEMS and Microelectronics, vol. 3893, pp. 494-500

8. Nguyen, T, \& Madadnia J, 2000a "Electrohydrodynamic (EHD) Effects On Nucleation and Detachment Off surfaces" presented in the $7^{\text {th }}$ Australasian heat and mass transfer conference, Townsville, 3-6 July.

9. Nguyen, T, \& Madadnia J, 2000b " Effects of Variable Electric Fields on the Behavior of Bubbles in the Nucleate Pool Boiling Regime" presented in the $7^{\text {th }}$ Australasian heat and mass transfer conference, Townsville, 3-6 July.

10. Ogata, J. and Yabe, A. 1991 "Augmentation of Nucleate Boiling Heat Transfer by Applying Electric Fields": EHD Behavior of Boiling Bubble", ASME/JSME Thermal Engineering Proceedings 3:41-46.

11. Ogata, J. and Yabe, A., 1993, "Augmentation of Boiling Heat Transfer by Utilizing the EHD Effect-EHD behavior of Boiling Bubbles and Heat Transfer Characteristics”. Int. J. of Heat and Mass Transfer Vol. 36, pp. 783-791.

12. Paul D. D. and Khalik S. I. 1983, " A Statistical Analysis of Saturated Nucleate Boiling Along A Heated Wire", Int. J. Heat Mass Transfer, Vol. 26 No. 4, 1983, pp. 509-519.

13. Seyed-Yagoobi, J and Bryan, J.E. (1999), "Enhancement of Heat Transfer and Mass Transport in Single-Phase and Two-Phase flows with Electrohydrodynamics", Advances In Heat Transfer, Vol. 33 PP 95-186.

14. Seyed-Yagoobi, J., Geppert, C. A., Geppert, L. M., 1996, "Electrohydrodynamically Enhanced Heat Transfer in Pool Boiling,"Transactions of ASME, Journal of Heat Transfer, Vol. 118, pp. 233-237.

15. Turnbull, R. J., 1968 (a,b), " Electroconvective instability with a stabilizing temperature gradient a) Theory, b) Experimental results" Phys. Fluids 11. 2597-2603. 
a) Constant electric field $=0$

Heat flux varied $\left(12.1,8,3.70 \mathrm{~kW} / \mathrm{m}^{2}\right)$

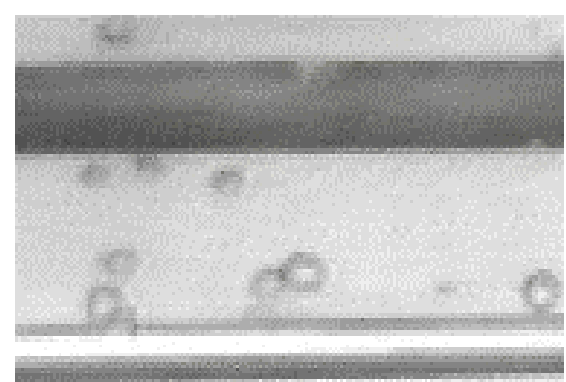

12.1
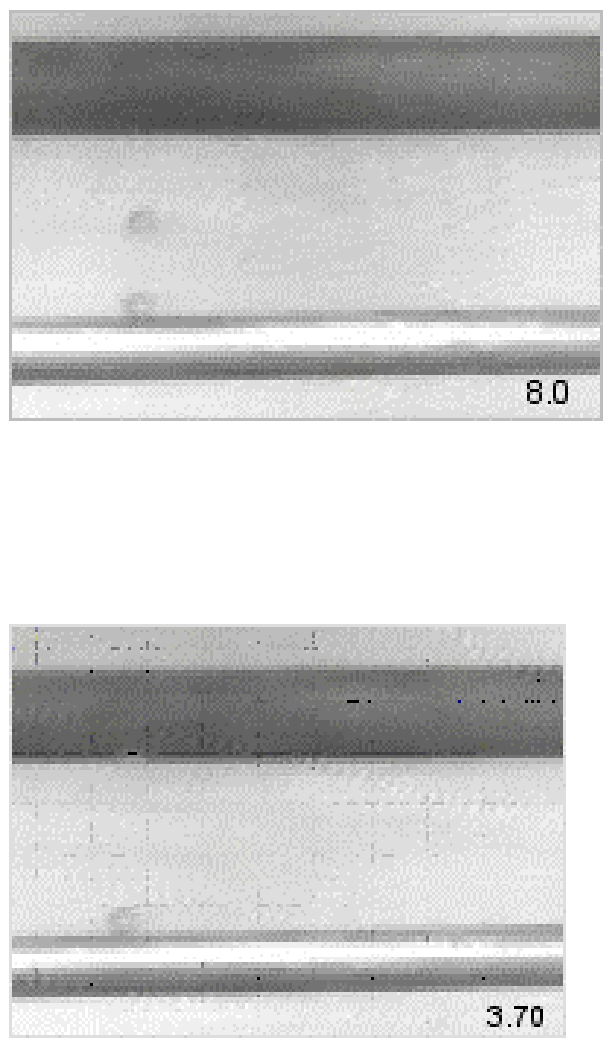

b) Constant heat flux $=35.4 \mathrm{~kW} / \mathrm{m}^{2}$

Electrode voltages varied $(6.0,6.6,7.5 \mathrm{kV})$
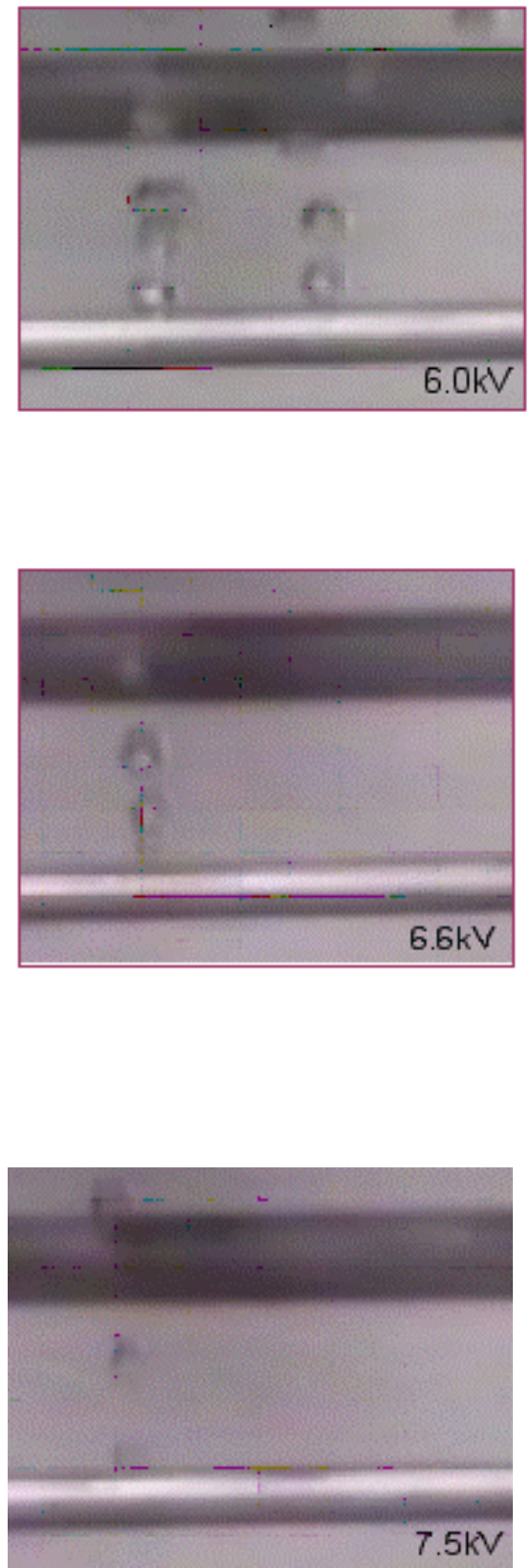


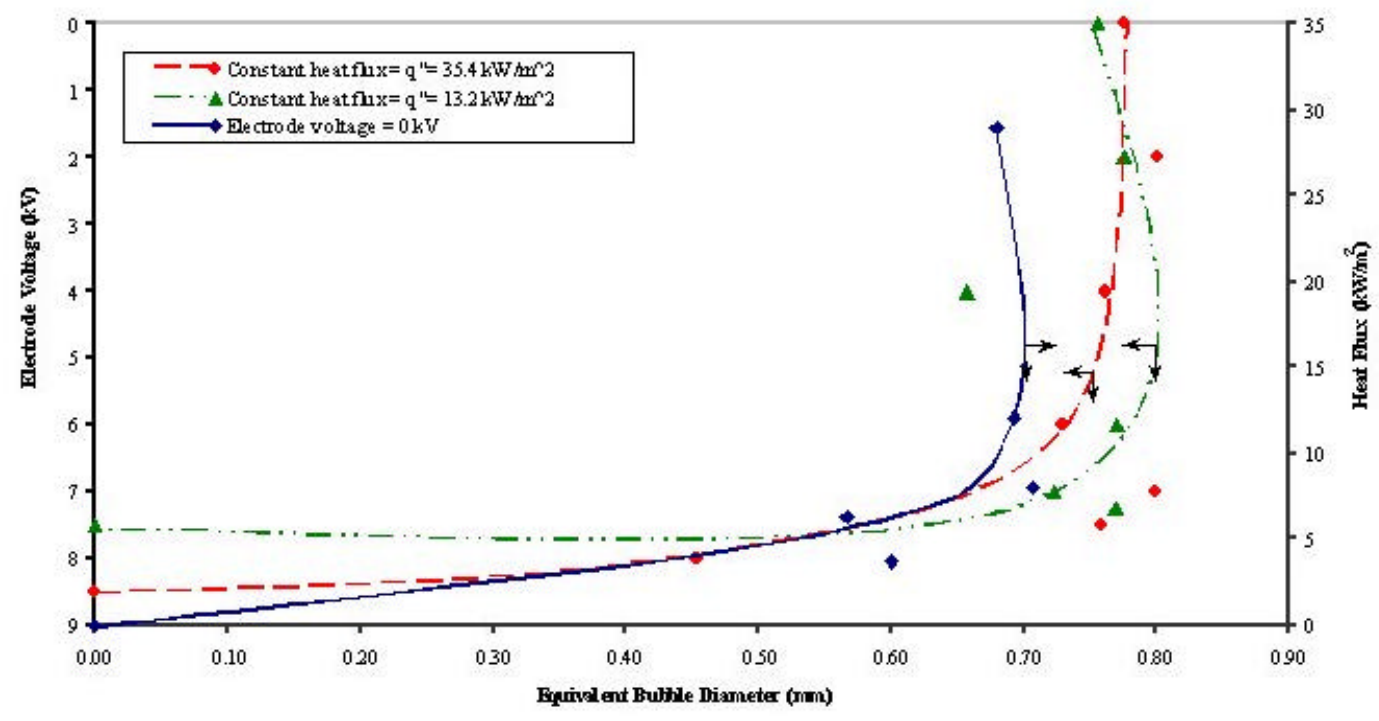

Figure 3. Effects of Electrode voltage and heat flux on bubble diameter

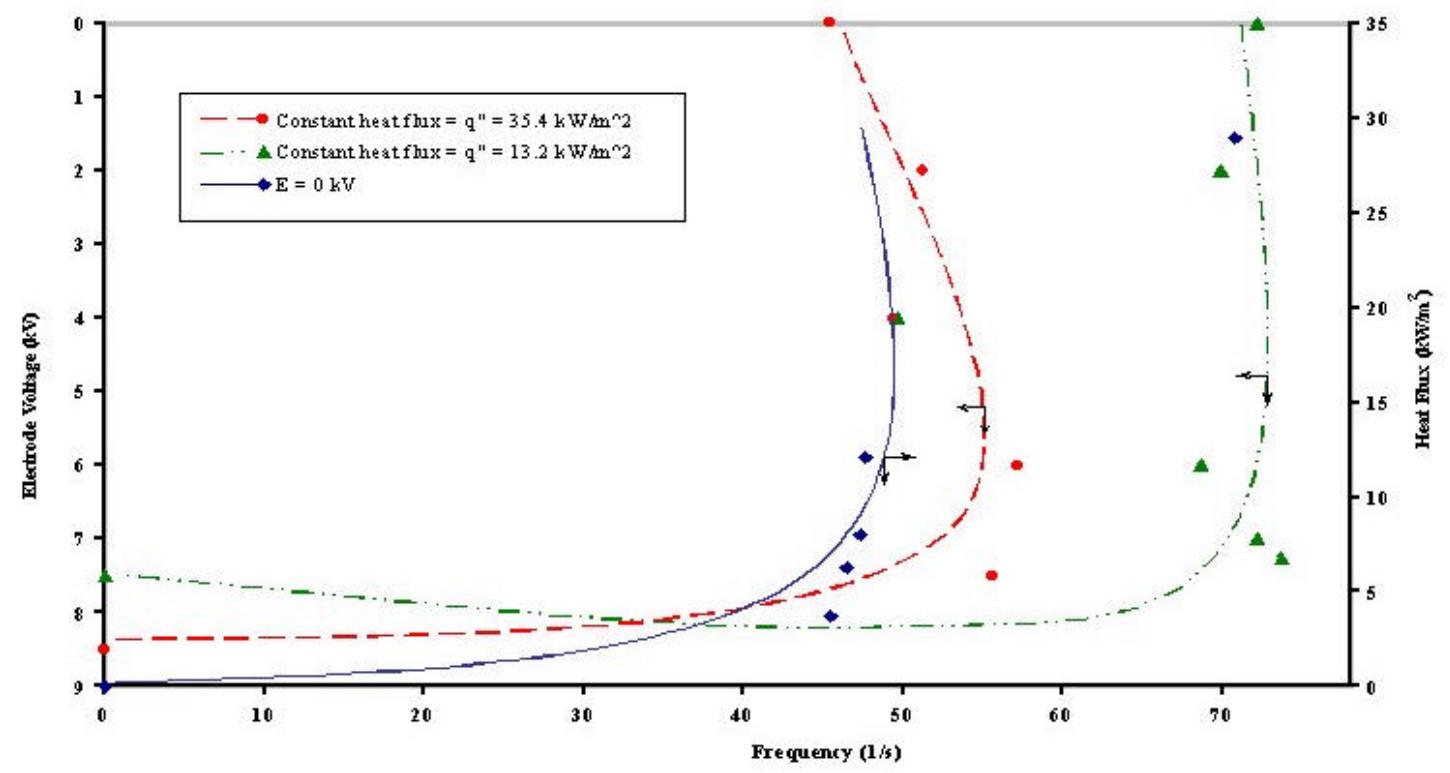

Figure 4. Effects of Electrode voltage and heat flux on frequency 


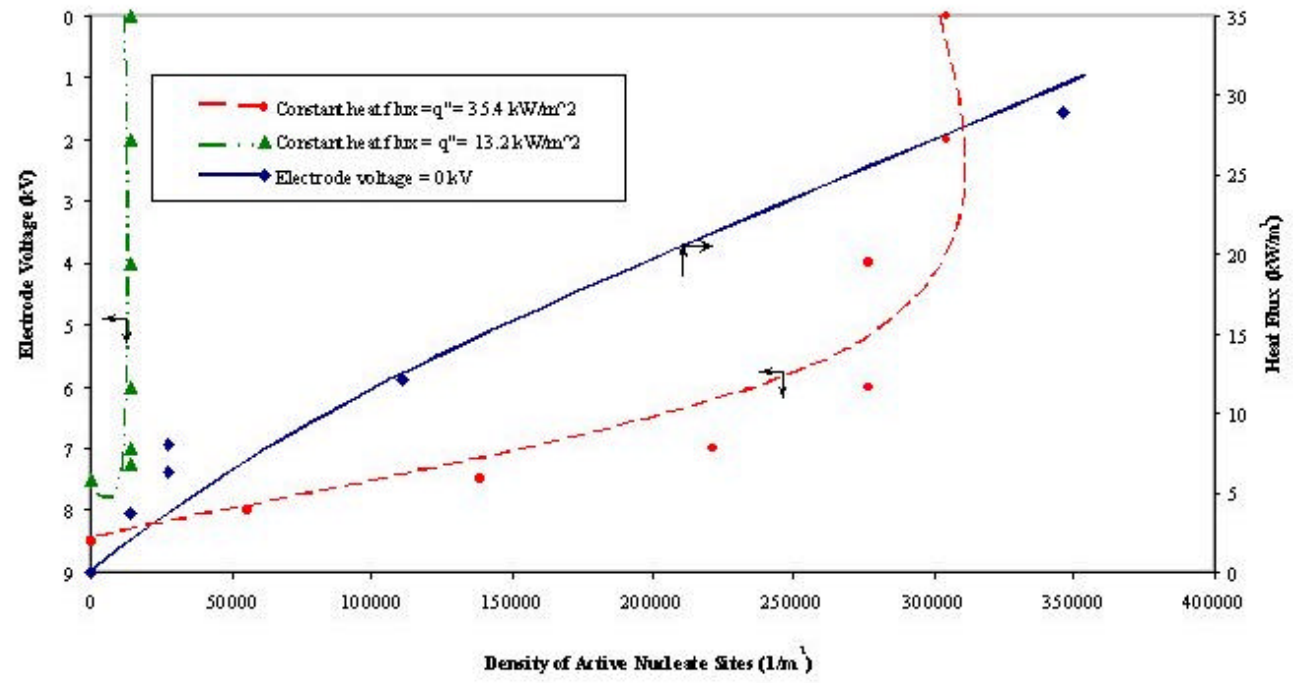

Figure 5. Effects of Electrode voltage and heat flux on the density of active nucleate sites

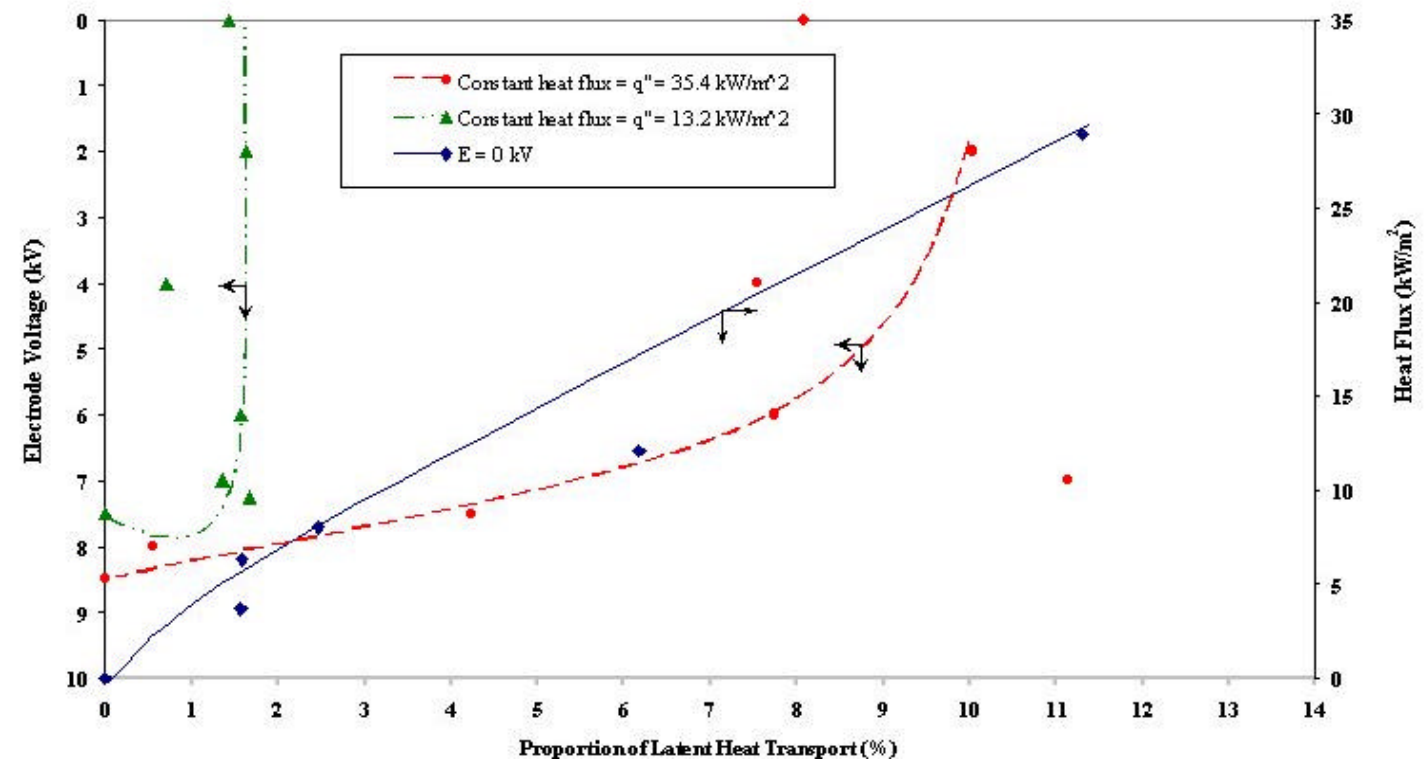

Figure 6. Effects of Electrode voltage and heat flux on the proportion of latent heat transport. 